\title{
Analysis of Coupling Degree of Economic Governance Capability and Ecological Governance Capability of Regional Industry
}

\author{
Hua Li, Wei He, Qiubai Sun* \\ School of Business Administration, University of Science and Technology Liaoning, Anshan, \\ China \\ *Corresponding Author.
}

\begin{abstract}
Promoting the modernization of the national governance system and governance capabilities is an important starting point to promote the advancement of the industrial structure and the green development of the industry, and it is also an inevitable requirement for my country to achieve high-quality economic development. The relationship between ecological governance capabilities and economic governance capabilities runs through national governance. A main line of the modernization of the system and governance capabilities, only by accurately measuring and evaluating my country's ecological and economic governance and the synergy between the two can we better promote the development of the new normal economy. For this reason, based on the research and evaluation of province data from 2015 to 2019, this paper found that the ecological governance capabilities and economic governance capabilities of China's provinces are quite different. Further, in terms of the coupling coordination degree between the two, in the time dimension, the coupling coordination degree between the ecological governance capacity and economic governance capacity of each province showed a trend of increasing and then decreasing during the period 2015-2019, but generally showed an upward trend, with the highest point being reached in 2016; in the regional dimension, there were large regional differences in the coupling coordination degree between the ecological governance capacity and economic governance capacity, the coupling coordination degree of ecological governance capacity and economic governance capacity is positively correlated with the level of economic development. This paper provides a theoretical basis for the objective evaluation of the coupling coordination degree of ecological and economic governance capacity in China, and has important practical significance for the coordinated promotion of the modernization of the national governance system and governance capacity.
\end{abstract}

Keywords: Economic governance capacity, ecological governance capacity, coupling coordination degree, time dimension, area dimension, regional industry

\section{Introduction}

In 2013, the Third Plenary Session of the 18th Central Committee proposed to promote the modernization of the national governance system and governance capacity. Under the situation where the institutional construction of the national governance system tends to be perfect, the national governance capacity is an urgent issue to be addressed by both political and academic circles on how to integrate economic development, political stability, cultural prosperity, social stability, ecological civilization and Party construction. As an important area of national governance, economic and ecological governance capacity has important research value. How to realize the combination of economic and ecological governance is an urgent issue for both the theoretical and practical communities, and is the main research content of this paper. The contradiction between China's economic development and the construction of ecological civilization was particularly prominent, before the modernization of the national governance system and governance capacity was proposed. especially because China's extensive development method has paid a huge environmental price, for example, between 2003 and 2011, although China's economy is growing rapidly, its energy consumption has increased by as much as $89.35 \%$ (Huang Maoxing and Lin Shoufu, 2013) [1], which is in contrast to Xi Jinping's proposal that "Lucid waters and lush mountains are

ISSN: 0010-8189

(C) CONVERTER 2020 
invaluable assets." Therefore, to strike a balance between economic development and ecological civilization, to treat the two as an organic unified whole and to promote them in a circular manner, is an important shift in the construction of ecological civilization and an important embodiment of high-quality economic development, and an important sign of the modernization of national governance capacity. The synergistic promotion of economic development and ecological civilization construction, in its connotation, is actually the synergistic integration of national economic governance capacity and ecological governance capacity, and the construction of regional governance capacity is the title of promoting the modernization of national governance capacity. For this reason, based on the economic development and ecological civilization construction data of 31 provinces in China from 2015 to 2019, this paper uses the entropy value method to synthesize the regional economic governance capacity and ecological governance capacity, and then the coupling coordination degree model is used to measure the coupling degree and coupling coordination degree of the economic governance capability and ecological governance capability of each province from 2015 to 2019, and relevant conclusions and policy recommendations were drawn.

\section{Literature Review}

With the orderly promotion of the modernization of the national governance system and governance capacity, many scholars have constructed comprehensive evaluation indicators for national governance capacity based on different principles and standards, including the construction of indicators of economic governance capacity and ecological governance capacity. In terms of economic governance capacity, Liu Chengli (2015) [2] first defines economic governance capacity as the government's ability to coordinate the overall economic development and regulate microeconomic subjects, and emphasizes that economic governance capacity is the main mean to implement the system; Tian Fa and Zhou Wuxing (2016) [3] based on provincial data from the perspective of public finance, constructed a comprehensive evaluation system covering the six indicators of infrastructure construction, welfare achievement distribution, and economic governance stability, efficiency, innovation, and sustainability. And found that the economic governance capabilities of various regions in my country have been steadily improved, but there are still certain differences among regions; Li Hua et al. (2019) [4] based on data from Liaoning province, used principal component analysis to comprehensively construct indicators of economic governance capacity in Liaoning province, and found that the economic governance capacity is generally on an upward trend.

In terms of ecological governance capacity, Li Xiaoxi et al. (2015) [5] first emphasized that coordinating the relationship between ecological civilization construction and economic growth is the key to modernizing ecological governance capacity, and pointed out that the government should clarify the relationship with the ruling party, the people's congress, society and the public, as well as further clarify the relationship between the same level of government and its parts. Zhang Lei (2015) [6] put forward the problem that the government still has poor ability to implement and respond to ecological governance in my country. The performance evaluation system should be further improved, and environmental issues should be governed in accordance with the law in an orderly manner; In order to effectively evaluate the government's ecological governance capacity, He Zhongliang and Li Qun (2019) [7] used AHP to construct a comprehensive evaluation index of the provincial government's ecological governance capacity based on data from Liaoning Province and compared it with the national average ecological governance capacity, and found that the ecological governance capacity of the Liaoning government changed from above the national average to below the national average.

Most of the above literature evaluates national governance capacity from a single dimension such as economic development and ecological civilization, but there is little literature on comprehensive analysis to evaluate the multidimensional integration and coordination of national governance capacity. Zhang Jundi (2018) [8] theoretically overview of promoting national governance system and governance modernization needs of economic, cultural, social, ecological, political party building collaborative governance and integration, the overall optimization of the six sub-system integration, and synergistically promoting the national governance capacity in order to ensure the adaptability, scientific and advanced nature of the national governance capacity, but does not

ISSN: 0010-8189 
quantify the degree of integration and synergistic promotion effect among the subsystems. At the same time, the established literature indicates that the coordinated coupling of economic development and ecological civilization is still difficult, and further transformation from rough economic development to high-quality development is still needed, therefore, it requires a comprehensive evaluation of the level of coordination of national economic governance capacity coupled with the ecological governance capability.

In summary, the marginal contribution of this paper is to quantify the degree of coupling coordination degree of the economic and ecological governance capacity of provincial governments based on a coupling coordination degree model after a comprehensive evaluation of the government's economic and ecological governance capacity, in order to provide a reference for further promoting national governance systems and governance modernization.

\section{Indicator selection and evaluation methodology}

\subsection{Selection of Indicators}

This paper refers to He Zhongliang and Li Qun's (2019) setting of the government's ecological governance capacity evaluation system, and makes corrections to select the green coverage rate of built-up areas (positive indicator)in 31 provinces across the country from 2015 to 2019, the harmless domestic waste treatment rate (positive indicator), the proportion of days with annual air quality reaching and better than level 2 (positive indicator), the city gas penetration rate (positive indicator), the economic governance capacity covers the following eight secondary indicators: the rate of the secondary industry in GDP (positive indicator), the rate of the tertiary industry in GDP (positive indicator), the per capita gross regional product (positive indicator), and the per capita disposable income (positive indicator), per capita disposable income (positive indicator), total retail sales of consumer goods (positive indicator), registered urban unemployment rate (negative indicator), foreign trade (positive indicator) and per capita consumption expenditure (positive indicator), among them, foreign trade is the ratio of the total import and export of goods to regional GDP. The above data are obtained from the "Statistical Yearbook"(2015-2019) of each province .

\subsection{Research Methodology}

This paper uses the entropy value method to construct comprehensive weight evaluation index, firstly homogenizing heterogeneous indicators through standardization, using the entropy value method to synthesize secondary indicators into primary indicators of ecological governance capacity and economic governance capacity, and finally the coupling and coordination degree model is used to calculate and analyze the coupling and coordination degree of the ecological governance capacity and economic governance capacity of each province from 2015 to 2019 .

\subsubsection{The Entropy Method}

(1) Data standardization

Let there be a total of $m$ provinces, $n$ secondary indicators and $x_{i j}$ denote the $j$ th secondary indicator for province $i$, where $i=1,2 \ldots m, j=1,2 \ldots n$, the normalization of the positive indicators is treated as:

$$
x_{i j}^{\prime}=\frac{x_{i j}-\min \left(x_{1 j}, x_{2 j} \cdots x_{m j}\right)}{\max \left(x_{1 j}, x_{2 j} \cdots x_{m j}\right)-\min \left(x_{1 j}, x_{2 j} \cdots x_{m j}\right)}
$$

The standard price treatment for negative indicators is as follows:

(2) Determine the weight of each secondary indicator:

$$
x_{i j}^{\prime}=\frac{x_{i j}-\min \left(x_{1 j}, x_{2 j} \cdots x_{m j}\right)}{\min \left(x_{1 j}, x_{2 j} \cdots x_{m j}\right)-\max \left(x_{1 j}, x_{2 j} \cdots x_{m j}\right)}
$$


(3) Calculate the entropy value of the $j t h$ indicator:

$$
p_{i j}=\frac{x_{i j}}{\sum_{i=1}^{m} x_{i j}}
$$

$$
e_{j}=-k \sum_{i=1}^{m} p_{i j} \ln \left(p_{i j}\right), \text { where } k=\frac{1}{\ln (m)}, m \text { is the total number of samples (4) }
$$

(4) Calculate the information utility value of the $j t h$ indicator:

$$
g_{j}=1-e_{j}
$$

(5) Calculate the weight of the jth indicator:

$$
w_{i j}=\frac{g_{j}}{\sum_{j=1}^{n}\left(g_{j}\right)}
$$

(6) Calculate the comprehensive score of each province's ecological governance capacity and economic governance capacity:

$$
S_{i}=\sum_{j=1}^{n} w_{j} \cdot p_{i j}
$$

\subsubsection{Coupling Coordination Degree Model}

The degree of coupling is designed to measure the magnitude of the coupling between the two subsystems and focuses on reflecting the degree of integration between the two subsystems, and does not reflect the extent to which the two systems promote each other. Therefore, this paper constructs a coupling model of ecological governance capacity and economic governance capacity, set as follows:

$$
C=\sqrt{\frac{H_{1} \times H_{2}}{\left(H_{1}+H_{2}\right)^{2}}}
$$

$\mathrm{C}$ represents the coupling degree of ecological governance capability and economic governance capability, $H_{1}$ represents ecological governance capability, $\mathrm{H}_{2}$ represents economic governance capability. The greater the coupling degree, the stronger the degree of mutual integration between ecological and economic governance capabilities, and because the coupling degree does not reflect the degree of coordination and promotion between ecological and economic governance capabilities, further this paper sets the coupling coordination degree model as follows:

$$
D=\sqrt{C \times T}, \quad T=a H_{1}+b H_{2}
$$

D denotes the coupling coordination degree of ecological governance capacity and economic governance capacity, similarly, $H_{1}$ denotes ecological governance capacity, $H_{2}$ denotes economic governance capacity, $\mathrm{T}$ is the comprehensive coordination index, $\mathrm{a}$ and $\mathrm{b}$ are the corresponding coefficients to be determined for ecological governance capacity and economic governance capacity, because in the orderly promotion of the modernization of the national governance system and governance capacity, ecological governance capacity and economic governance capacity are equally important, therefore let $\mathrm{a}=\mathrm{b}=0.5$, at this point, the greater the coupling coordination degree between ecological governance capacity and economic governance capacity, indicating that the province's ecological governance capacity and economic governance capacity are more synergistic and circularly promoted. Drawing on the level of coupling coordination degree established by Zhu Jianhua et al ${ }^{[9]}$, the settings in this paper are shown in Table 1.

Table 1 Criteria for the classification of coupling coordination levels 


\begin{tabular}{|c|c|}
\hline Coupling coordination & Coupling coordination level \\
\hline $0.8<\mathrm{D} \leq 1$ & Extremely coupled coordination \\
\hline $0.6<\mathrm{D} \leq 0.8$ & Highly coupled coordination \\
\hline $0.5<\mathrm{D} \leq 0.6$ & Moderate coupling coordination \\
\hline $0.4<\mathrm{D} \leq 0.5$ & Primary coupling coordination \\
\hline $0.3<\mathrm{D} \leq 0.4$ & Low coupling coordination \\
\hline $0<\mathrm{D} \leq 0.3$ & Very low coupling coordination \\
\hline
\end{tabular}

\section{Results and Discussion}

\subsection{Indicator Weighting Results}

In this paper, the entropy method is used to synthesize the secondary indicators into the primary indicators of ecological governance capacity and economic governance capacity for comprehensive evaluation. By calculating the weights of the corresponding indicators after dimensionless processing of the original data for the assessment of the ecological governance capacity and economic governance capacity of local governments, the scores of the ecological governance capabilities and economic governance capabilities of the local governments pointed to by the indicators can be calculated, so as to evaluate the relative strength and order of local governments' governance capacity under the same dimensional indicators. The directions and weights of the indicators are shown in Table 2 , which shows that among the ecological governance capacity, those with greater weight include the harmless disposal rate of domestic waste and the number of environmental emergencies. As a major problem in degradation management, domestic waste has always been a chronic problem to improve the ability of ecological governance. How to treat domestic waste more environmentally still needs to be further studied, and environmental emergencies are becoming more and more important as an indicator for evaluating the comprehensive ecological governance capabilities of various regions. It is more able to scientifically evaluate the prevention and control standards of the daily ecological management of the area. In terms of economic governance capacity, foreign trade accounts for the highest proportion, indicating that international trade has a significant impact on economic governance capacity, while the total retail sales of consumer goods is second only to foreign trade, which also coincides with the current advocacy of "gradually forming a new development pattern with domestic and international cycles as the main body and mutual promotion of domestic and international double cycles". Further effectively stimulating the potential of domestic consumption and promoting the coordinated promotion of internal and external economic cycles are important signs of improving economic governance capabilities. The proportion of tertiary industry in GDP is much higher than the weight of the second industries in GDP, indicating that advanced industrial structure can better demonstrate economic governance capabilities.

Table 2 Results of the calculation of the weights of the indicators of ecological and economic governance capacity

\begin{tabular}{|c|c|c|c|}
\hline Tier 1 indicators & Secondary indicators & Direction & Weighting \\
\hline \multirow{8}{*}{$\begin{array}{l}\text { Ecological } \\
\text { governance capacity }\end{array}$} & Greenery coverage in built-up areas & + & $2.90 \%$ \\
\hline & Harmless disposal rate of domestic waste & + & $28.49 \%$ \\
\hline & $\begin{array}{c}\text { Proportion of days with annual air quality at } \\
\text { or better than Class } 2\end{array}$ & + & $6.65 \%$ \\
\hline & City gas penetration rate & + & $12.53 \%$ \\
\hline & $\begin{array}{l}\text { Investment completed in industrial pollution } \\
\text { control }\end{array}$ & + & $17.89 \%$ \\
\hline & Forest cover & + & $10.10 \%$ \\
\hline & Number of environmental emergencies & - & $21.32 \%$ \\
\hline & Share of crop area sown & + & $11.39 \%$ \\
\hline
\end{tabular}

ISSN: 0010-8189 


\begin{tabular}{|c|c|c|c|}
\hline \multirow{5}{*}{$\begin{array}{c}\text { Economic } \\
\text { governance capacity }\end{array}$} & Share of secondary sector in GDP & + & $2.90 \%$ \\
\cline { 2 - 4 } & Tertiary sector as a share of GDP & + & $10.54 \%$ \\
\cline { 2 - 4 } & Gross regional product per capita & + & $6.47 \%$ \\
\cline { 2 - 4 } & Dotal retail sales of social consumer goods & + & $11.80 \%$ \\
\cline { 2 - 4 } & Registered urban unemployment rate & - & $3.26 \%$ \\
\cline { 2 - 4 } & Foreign trade & + & $40.21 \%$ \\
\cline { 2 - 4 } & Per capita consumption expenditure & + & $9.16 \%$ \\
\hline
\end{tabular}

\subsection{Analysis of Governance Capacity}

To facilitate comparison and the calculation of the coupled coordination model below, this paper standardizes the comprehensive evaluation index of ecological governance capacity and economic governance capacity of each province, and sets the value range between 0 and 1 . Due to space limitations, this article only lists the comprehensive evaluation index of the ecological governance capacity and economic governance capacity of each province in 2019 and the national average. The results are shown in Table 3, it can be seen that regardless of the ecological governance capacity or the economic governance capacity, there are huge differences among the provinces. The highest ecological governance capacity is 0.78 in Shandong Province, which is much higher than the national average of 0.23 , followed by Jiangsu Province and Guangdong Province, while the lowest is in the Tibet Autonomous Region and Heilongjiang Province; the highest economic governance capacity is in Shanghai, followed by Jiangsu Province and Guangdong Province, while the lowest is in the Tibet Autonomous Region. Jiangsu, Guangdong and Tibet Autonomous Regions have the same ranking clusters in terms of ecological and economic governance capabilities. In terms of their values, the ecological and economic governance capacities of Jiangsu Province are 0.53 and 0.45 respectively, while the ecological and economic governance capacities of Guangdong Province are 0.47 and 0.44 respectively, which is not a big difference between the two governance capacities, or there is a certain coupling coordination degree relationship. For this reason, the coupling coordination degree analysis is carried out below.

Table 3 Evaluation of ecological governance capacity and economic governance capacity in 2019

\begin{tabular}{|c|c|c|c|c|c|c|c|c|}
\hline Province & Ecology & Economy & Province & Ecology & Economy & Province & Ecology & Economy \\
\hline Shanghai & 0.22 & 0.59 & Guangdong & 0.47 & 0.44 & Gansu & 0.18 & 0.05 \\
\hline Yunnan & 0.09 & 0.11 & Guangxi & 0.08 & 0.11 & Fujian & 0.18 & 0.32 \\
\hline $\begin{array}{c}\text { Inner } \\
\text { Mongolia }\end{array}$ & 0.21 & 0.15 & Xinjiang & 0.15 & 0.10 & Tibet & 0.00 & 0.06 \\
\hline Beijing & 0.16 & 0.58 & Jiangsu & 0.53 & 0.45 & Guizhou & 0.14 & 0.08 \\
\hline Jilin & 0.05 & 0.09 & Jiangxi & 0.22 & 0.14 & Liaoning & 0.18 & 0.18 \\
\hline Sichuan & 0.31 & 0.19 & Hebei & 0.31 & 0.14 & Chongqing & 0.06 & 0.21 \\
\hline Tianjin & 0.11 & 0.29 & Henan & 0.41 & 0.18 & Shaanxi & 0.45 & 0.15 \\
\hline Ningxia & 0.09 & 0.10 & Zhejiang & 0.34 & 0.43 & Qinghai & 0.05 & 0.07 \\
\hline Anhui & 0.26 & 0.18 & Hainan & 0.01 & 0.12 & Heilongjiang & 0.03 & 0.09 \\
\hline Shandong & 0.78 & 0.28 & Hubei & 0.27 & 0.23 & Hunan & 0.26 & 0.18 \\
\hline Shanxi & 0.45 & 0.10 & & & & National & & \\
average & 0.23 & 0.21 \\
\hline
\end{tabular}

4.3 Coupling Coordination Degree Analysis 
In order to verify whether there is a synergistic effect between ecological governance capacity and economic governance capacity, that is, a coupling coordination degree relationship, this paper calculates the coupling coordination degree of each province during 2015-2019, and the results are shown in Table 4, which show that there is indeed a coupling coordination degree relationship between ecological governance capacity and economic governance capacity to different degrees in different years in each province.

In terms of the time dimension, the following phenomenon exists across the country and even in the vast majority of provinces, where the coupled coordination of ecological and economic governance capacity increased from 2015 to 2016, while decreasing to varying degrees from 2016 to 2019, with the national average of coupled coordination rising from 0.28 to 0.33 from 2015 to 2016, up from very low coupling coordination to low coupling coordination standards, but declined to 0.30 by 2019, with the largest decline between 2016 and 2017, probably due to the official implementation of the new Environmental Protection Law in 2015 and the fact that the Ministry of Environmental Protection directly "interviewed" and "charged" the top leaders of local governments, leading to the fact that in 2016, local governments were vigorously developing the economy while taking into account ecological and environmental protection issues. However, the 'heat' did not continue effectively, resulting in a sharp decline in the coupling coordination degree of ecological and economic governance capacity across the provinces from 2016 to 2017. After 2017, there has been differentiation in various regions. Shandong Province, Heilongjiang Province, Liaoning Province, etc. continued to decline, while Jiangsu Province, Zhejiang Province, Guizhou Province and other places experienced a rebound. The possible reason is the problem of industrial structure. Shandong Province and Liaoning Province are still dominated by the secondary industry in industrial economy. Energy intensity remains high, and pollution emissions have always been a problem in governance. With the relaxation of environmental protection inspections, local governments are more inclined to develop the economy. While Jiangsu Province and Zhejiang Province are mainly service industries, the tertiary industry is well developed, and the relaxation of environmental regulation has little impact on the overall economic development and environmental protection.

From the regional dimension, the coupling coordination degree of ecological and economic governance capacity varies greatly among provinces. In 2015, four provinces, including Shandong, Jiangsu, Guangdong and Zhejiang, reached the primary coupling coordination degree standard, eight provinces and cities, including Shanghai and Beijing, reached the low coupling coordination degree standard, and the rest of the provinces and cities were at the very low coupling coordination degree standard; in 2016, the coupling coordination degree of ecological and economic governance capacity basically reached its peak everywhere, at which time the highest was Shanghai at 0.57, and four other provinces and cities, including Shandong and Jiangsu, met the medium coupling coordination standard, two provinces and cities, including Beijing and Hubei, met the primary coupling standard, eight provinces and cities, including Inner Mongolia, Sichuan and Tianjin, met the low coupling coordination standard, and the rest were very low coupling coordination standards; while in 2019, five provinces and cities, including Shanghai, Shandong, Jiangsu, Guangdong and Zhejiang, met the primary coupling coordination standard, 10 provinces and cities such as Beijing and Sichuan meet the low coupling coordination standard, and the rest are still very low coupling coordination standards. It can be found that the coupling coordination between ecological governance capacity and economic governance capacity is higher in the large economic provinces such as Guangdong, Jiangsu and Shandong, and lower in the weak economic provinces such as Tibet and Heilongjiang, which is related to the GDP. The possible reason is that provinces with a lower level of economic development are more focused on economic growth, and investment funds for ecological governance are limited, and the economic governance capacity of local governments is higher than that of ecological governance, resulting in a lower degree of coupling and coordination between the two, and a lower level of economic development. Higher provinces, On the one hand, provinces with higher levels of economic development are facilitated by economic development and have more investment funds available for pollution control, thus ensuring the ecological governance capacity of local governments, ecological governance capacity benefits from economic governance capacity, and economic governance capacity provides financial guarantees for ecological governance capacity. On the other hand, good ecological governance capacity is a soft power to enhance economic governance capacity, and the optimization of the ecological environment can stimulate the tourism market and boost local consumption and investment in many

ISSN: 0010-8189 
ways, thus improving economic governance capacity. Therefore, ecological governance capability and economic governance capability complement each other, integrate with each other, and promote each other. Only by giving full play to the synergy between the two can we better promote the modernization of the national governance system and capacity, achieve the upgrading of the industrial structure and optimization of the ecological environment, and ultimately realize that "Lucid waters and lush mountains are invaluable assets".

Table 4 Analysis of coupling coordination by province, 2015-2019

\begin{tabular}{|c|c|c|c|c|c|c|c|c|c|c|c|}
\hline Province & 2015 & 2016 & 2017 & 2018 & 2019 & Province & 2015 & 2016 & 2017 & 2018 & 2019 \\
\hline Shanghai & 0.36 & 0.57 & 0.45 & 0.44 & 0.43 & Jiangxi & 0.23 & 0.27 & 0.24 & 0.26 & 0.30 \\
\hline Yunnan & 0.20 & 0.22 & 0.18 & 0.19 & 0.22 & Hebei & 0.32 & 0.30 & 0.30 & 0.31 & 0.32 \\
\hline Inner Mongolia & 0.32 & 0.33 & 0.32 & 0.31 & 0.30 & Henan & 0.30 & 0.39 & 0.35 & 0.36 & 0.37 \\
\hline Beijing & 0.38 & 0.44 & 0.40 & 0.45 & 0.39 & Zhejiang & 0.46 & 0.54 & 0.43 & 0.43 & 0.44 \\
\hline Jilin & 0.21 & 0.24 & 0.21 & 0.22 & 0.19 & Hainan & 0.15 & 0.22 & 0.19 & 0.16 & 0.13 \\
\hline Sichuan & 0.26 & 0.32 & 0.30 & 0.32 & 0.35 & Hubei & 0.28 & 0.41 & 0.33 & 0.34 & 0.35 \\
\hline Tianjin & 0.33 & 0.31 & 0.26 & 0.29 & 0.30 & Hunan & 0.30 & 0.28 & 0.29 & 0.33 & 0.33 \\
\hline Ningxia & 0.18 & 0.27 & 0.23 & 0.27 & 0.22 & Gansu & 0.14 & 0.21 & 0.16 & 0.17 & 0.22 \\
\hline Anhui & 0.25 & 0.34 & 0.28 & 0.30 & 0.33 & Fujian & 0.39 & 0.40 & 0.35 & 0.34 & 0.34 \\
\hline Shandong & 0.45 & 0.55 & 0.50 & 0.48 & 0.48 & Tibet & 0.00 & 0.00 & 0.04 & 0.05 & 0.06 \\
\hline Shanxi & 0.23 & 0.31 & 0.32 & 0.32 & 0.32 & Guizhou & 0.16 & 0.20 & 0.20 & 0.21 & 0.23 \\
\hline Guangdong & 0.45 & 0.53 & 0.52 & 0.50 & 0.48 & Liaoning & 0.32 & 0.36 & 0.32 & 0.30 & 0.30 \\
\hline Guangxi & 0.25 & 0.29 & 0.23 & 0.24 & 0.21 & Chongqing & 0.25 & 0.30 & 0.28 & 0.26 & 0.23 \\
\hline Xinjiang & 0.21 & 0.28 & 0.24 & 0.22 & 0.25 & Shaanxi & 0.34 & 0.37 & 0.33 & 0.35 & 0.36 \\
\hline Jiangsu & 0.48 & 0.56 & 0.44 & 0.46 & 0.49 & Qinghai & 0.15 & 0.20 & 0.15 & 0.15 & 0.17 \\
\hline Heilongjiang & 0.22 & 0.26 & 0.20 & 0.18 & 0.16 & National & 0.28 & 0.33 & 0.29 & 0.30 & 0.30 \\
\hline
\end{tabular}

\section{Conclusion and Suggestions}

Dealing with ecological governance capacity and economic governance capacity is a main thread running through the modernization of the national governance system and governance capacity. Only by accurately measuring and evaluating China's ecological and economic governance capabilities and the synergy between the two can we better promote the new normal economic development. After conducting research and evaluation based on provincial data from 2015 to 2019, it is found that the ecological and economic governance capabilities vary greatly between provinces in China. In terms of the coupling coordination degree of the two, in the time dimension, the coupling coordination degree of ecological and economic governance capacity of each province showed a trend of increasing and then decreasing between 2015 and 2019, but the overall trend was on the rise, with the highest point reached in 2016; in the regional dimension, there were large regional differences in the coupling coordination degree of ecological and economic governance capacity, with Guangdong, Jiangsu and Shandong, with higher economic development level have a higher degree of coupling and coordination, while Tibet and Heilongjiang, with lower economic development level have a lower degree of coupling coordination degree, the characteristic fact that the degree of coupling coordination between ecological governance capacity and economic governance capacity appears to be positively correlated with the level of economic development.

Therefore, the policy recommendations of this paper are as follows: (1) Correctly understand the coupling coordination degree between ecological and economic governance capacity, governments at all levels should coordinate the promotion of economic development and ecological governance, to avoid the more costly and sloppy development path, we must deal with the relationship between governments at all levels and departments at the same level, to eliminate the environmental responsibility hole and put ecological governance into legal system construction. (2) Continuously and effectively promote environmental regulation, do not adopt a broad-brush approach, let alone neglect regulation, and gradually improve the strength of environmental regulation, thereby

ISSN: 0010-8189 
improving the coupling coordination degree of ecological and economic governance. (3) Deal with regional differences in the degree of coupling coordination degree between ecological and economic governance, and guide provinces with a low degree of coupling coordination degree to strengthen their understanding, reasonably determine their economic development goals, and integrate ecological governance into economic development.

\section{Acknowledgements}

This research was supported by the Natural Science Foundation of China (71771112, 71571091), Innovative Talents Project of Liaoning Province (WCR2018003), Natural Science Foundation of Liaoning Province of China (20180550274), University of Science and Technology Liaoning Talent Project Grants (601011507-03), Project of Liaoning Provincial Federation Social Science Circles of China(L20BGL047), and Soft Science Project of Department of Science \&Technology of Liaoning Province of China(2020JH4/10100008) .

\section{References}

[1] M.X. Huang, S.F. Lin, "Pollution damage, environmental management and sustainable economic growth -- an analysis based on a five-sector endogenous economic growth model," Economic Research, vol.48, no.12, pp.30-41, 2013.

[2] C.L. Liu, "Modernization of economic governance system and governance capacity: A dual perspective of government and market," The Economist, no.05, pp.28-34, 2015.

[3] F. Tian, W.X. Zhou, "The construction and measurement of economic governance capacity indicator system--based on the perspective of public finance," Journal of Xi'an University of Finance and Economics, vol.29, no.03, pp.11-17, 2016.

[4] H. Li, Y. Wang, Q.B. Sun, "Evaluation of local government economic governance capacity: empirical evidence based on Liaoning Province," Statistics and Decision Making, vol.35, no.10, pp. 94-97, 2019.

[5] X.X. Li, Z. Zhao, W.F. Li, "Four major relationships for improving the modernization of national ecological governance system and governance capacity--an analysis based on field research and micro data," Management World, no. 05, pp.1-5, 2015.

[6] L. Zhang, "The ecological dimension of the modernization of national governance capacity - the perspective of the government's ecological governance capacity." Administrative Forum, vol. 22, no. 06, pp. 9-13, 2015.

[7] Z.L. He, Q. Li, "Research on the ecological governance evaluation index system based on AHP - taking Liaoning Province as an example," Practice and understanding of mathematics, vol. 49, no. 22, pp. 106-113, 2019.

[8] J.D. Zhang, "System construction, functional coupling and national governance system optimization - a complexity framework," Academic Research, no.09, pp.61-65, 2018.

[9] J.H. Zhu, H.J. Wang, P. Zheng, "Study on the coupled and coordinated development of circular economy and green finance in Guizhou Province," Economic Geography, vol.39, no.12, pp.119-128, 2019. 